\title{
Slope of Autocorrelation Function of Detrended 24-Hour RR-Intervals Independently Predicts Mortality in Postinfarction Patients
}

\author{
A Berkowitsch ${ }^{1}$, A Bauer ${ }^{2}$, R Schneider ${ }^{2}$, P Barthel $^{2}$, H Greiss $^{1}$, \\ HJ Schneider ${ }^{1}$, HF Pitschner ${ }^{1}$, G Schmidt ${ }^{2}$ \\ ${ }^{1}$ Kerckhoff-Klinik, Bad Nauheim, Germany \\ ${ }^{2}$ Medizinische Klinik der Technischen Universität München, Munich, Germany
}

\begin{abstract}
Although Heart Rate Variability (HRV) was shown to be associated with mortality of post myocardial infarction (MI) patients, the clinical use of conventional HRV parameters is limited. We proposed new method based on the analysis of autocorrelation function (ACF) of detrended $R R$ interval recordings. One thousand four hundred fifty five consecutive post MI patients were enrolled into the prospective blinded study at German Heart Center in Munich. The RR intervals were extracted from $24 \mathrm{~h}$ Holter recordings using integrated software.

The linear trend was estimated and subtracted from $R R$ recordings. The $A C F(m)$ was calculated for original and detrended RR-intervals. The slope $(A S)$ of regression line fitted for ACF $(m ; m=1,10)$ was dichotomized at AS=-0.0025. AS >-0.0025 was considered as pathological. The significant association of pathological AS was revealed in univariate analysis (Log Rank $=39.19, p<0.0001)$ and was confirmed in multivariate Cox-Model $(p<0.001)$.
\end{abstract}

\section{Introduction}

Although in several previous studies [1-3] depressed Heart Rate Variability (HRV) was shown to be associated with mortality of post myocardial infarction (MI) patients, the clinical use of conventional HRV parameters is limited. In the recent studies [4] performed on post MI patients receiving optimized $\mathrm{BB}$ medication only nonlinear HRV parameters such as power-low slope $(\beta)$ and short-term fractal exponent $(\alpha)$ were revealed to be independent predictors for mortality after adjustment of clinical covariates. The last parameter was defined using detrended fluctuation analysis (DFA). This method was derived from non-linear dynamics and quantifies the fractal scaling properties of RR fluctuations.

Two commonly used approaches for visualization of heart rate dynamics are autocorrelation function (ACF) and Poincaré plot (PP) construction were previously regarded as qualitative methods [6].
Recently proposed descriptors of PP based on fitting an ellipse to the plot [7] were also not confirmed to be independent predictors for mortality [4]. In another study PP was quantified using Pearson correlation coefficient $\left(r_{R R}\right)$ between $R_{n}$ and $R_{n+1}$ [5]. The sequence of correlation coefficients of lagged $P P\left(R R_{n} v s . R_{n+m}\right)$ is autocorrelation function $(\mathrm{ACF}(\mathrm{m}))$ of the lag $\mathrm{m}$. The prognostic value of rRR and of the pattern of ACF for clinical outcome have not been yet evaluated. Recently it was shown that original RR sequence can be regarded as a sum of stationary signal and trend [4].

For estimation and removal of trend we used an adaptive procedure based on calculation of the instantaneous signal mean. Distinctly from DFA reported in studies [4] this method is linear. The algorithm is in details described elsewhere [6]. We observed that difference in PP and in ACF pattern of detrended fluctuations between low risk and high risk patients is more pronounced (Fig 1). We propose to characterize PP by (rRR) and ACF by slope of regression line fitted for $\mathrm{ACF}(\mathrm{m})$ by $\mathrm{m}$ varying from $\mathrm{m}=1$ to $\mathrm{m}=10$ (Fig 1 ). This parameter was termed Autocorrelation Slope (AS). Aim of this study is to analyze prognostic value of ACF parameters of original RRI and of detrended fluctuations for late mortality in postinfarction patients.

\section{Methods}

One thousand four hundred fifty five consecutive post MI patients of either sex younger than 76 years of age were enrolled in the study at German Heart Center in Munich if they had survived an acute MI within the last 4 weeks and if they presented with sinus rhythm. An infarction was diagnosed if a patient had at least 2 of the following findings: chest pain for $>20$ minutes, creatine kinase $>200 \mathrm{U} / \mathrm{L}$, and ST-segment elevation of $>0.1 \mathrm{mV}$ in 2 or more limb leads or $>0.2 \mathrm{mV}$ in 2 or more contiguous pericardial leads at the time of admission. The institutional ethics committee approved the study protocol.

The 24-hour Holter recordings were processed by an Oxford Excel Holter system (Oxford Instruments) or by a 
Pathfinder 700 (Reynolds Medical). The tachograms were extracted using integrated into applied Holter system software. Prior to signal processing instantaneous mean value was estimated using algorithm described in $[10,11]$. The RR interval interpreted as artifacts or those longer than $2000 \mathrm{~ms}$ or shorter than $300 \mathrm{~ms}$ were replaced by instantaneous mean calculated for preceding sinus rhythm beat.

The PP and ACF were analyzed for original RR recordings $\left(\mathrm{r}_{\mathrm{RR}} 1 ; \mathrm{AS} 1\right)$ and detrended fluctuations $\left(\mathrm{r}_{\mathrm{RR}} 2\right.$, AS2). Based on observations in small test collective (20 pts) the cut-offs of rRR were pre-specified as $\mathrm{rRR} 1 \leq 0.5$ and $r_{R R} 1>0.5$ for original RRI recording and as $r_{R R} 2 \leq 0$ and $r_{R R} 2>0$ for detrended fluctuations. cut-offs of AS was pre-specified as AS1<-0.0025 and AS1 $\geq-0.0025$ for original RRI and as AS2<-0.02 and AS2 $\geq-0.02$ for detrended fluctuations. AS $\geq$ cut-offs value and $r_{R R} \leq$ cutoffs value were considered as abnormal. Following other risk predictors were prospectively selected: age of the patient, history of previous myocardial infarction, left ventricular ejection fraction (LVEF), presence of diabetes mellitus, mean heart rate, time domain heart rate variability parameters (SDNN,SDANN, RMSSD triangular index (HRVI), arrhythmia sign on Holter and fractal scaling exponent $\alpha$.

The cutoff points were prospectively defined as follows: age of the patient $<65$ and $\geq 65$ years, $\mathrm{LVEF} \leq 30 \%$ and $\mathrm{LVEF}>30 \%$, mean heart rate $<75 \mathrm{bpm}$ and $\geq 75 \mathrm{bpm}$, HRVI $\leq 20 \mathrm{U}$ and $>20 \mathrm{U}$, SDNN $\leq 65 \mathrm{~ms}$ and $>65 \mathrm{~ms}$; RMSSD $\leq 19 \mathrm{~ms}$ and $>19 \mathrm{~ms}$, SDANN $\leq 63$ $\mathrm{ms}$ and $>63 \mathrm{~ms}$, single VPBs $<10 \mathrm{VPBs}$ and $\geq 10 \mathrm{VPBs}$ per hour, and nonsustained ventricular tachycardia(VT) $<1$ run and $\geq 1$ run per 24 hours. The latter 2 parameters were used to form 2 arrhythmia categories (ARR), negative $(<10$ VPBs per hour and no nonsustained VT) and positive ( $>=10 \mathrm{VPBs} / \mathrm{h}$ or $\geq 1$ nonsustained VT in 24 hours). The fractal scaling exponent $\alpha$ was dichotomized as $\alpha \leq 0.65$ and $\alpha>0.65$. All risk predictors were measured during the second week after the index infarction.

\section{Results}

Out of 1455 patients 70 reached endpoint. The survival curves are presented at the figure 3 . As depicted at the table 1 the strongest predictor was $\mathrm{LVEF}<=30 \%$ providing hazard ratio of 7.1. All ACF parameters. calculated for both original RR intervals and detrended fluctuations shown significant association with death with hazard ratio $>4$ whereas conventional Holter parameters provided hazard ratio <3.0. The combination of ACF parameters computed for detrended RR intervals with one another and their combination with positive arrhythmia on Holter provided hazard ratio $>6.0$. Controversially to rRR2 the rRR1 was not revealed. In the multivariate
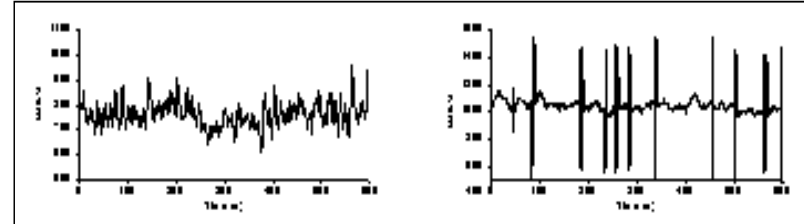

Original RRI (10 min)

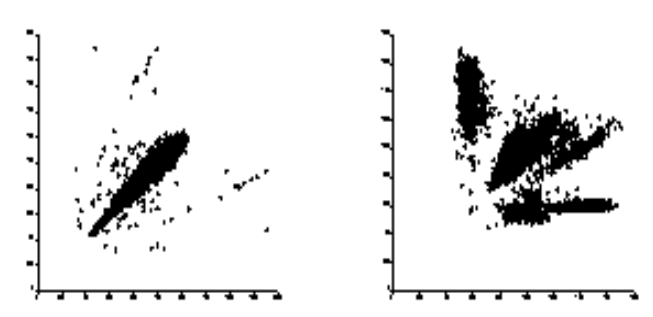

PP of original RRI (24h)
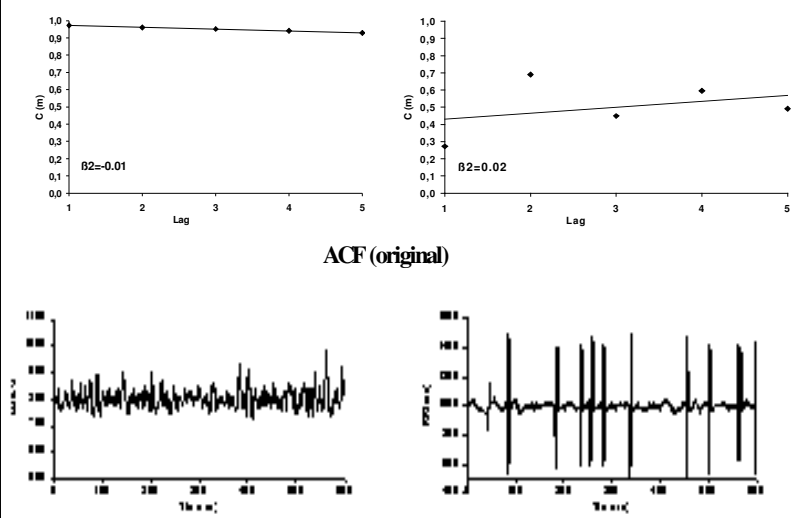

Detrended RRI (10 min)
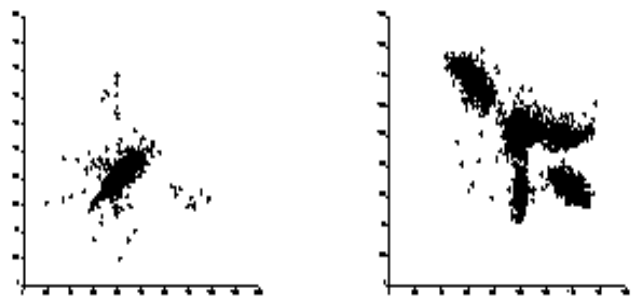

PP of detrended RRI (24h)

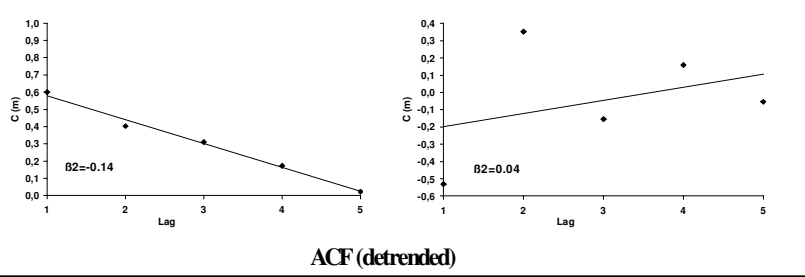

Figure 1: Left column- normal ACF in AMI survivor; right -abnormal ACF; patient died. The subtraction of trend from original RRI overlapped by frequent ventricular ectopy (right) provided changing of orientation of PP and negative correlation between $R_{n}$ and $\mathrm{RR}_{\mathrm{n}+1}$. 
analysis $\mathrm{LVEF}<30 \%$ was revealed to be strongest predictor of mortality after adjustment of clinical variables, the second strongest predictor was AS1. The predictive value of ACF parameters improved if combined with positive arrhythmia on Holter (table 1).

Table 1. Univariate and multivariate predictors of death.

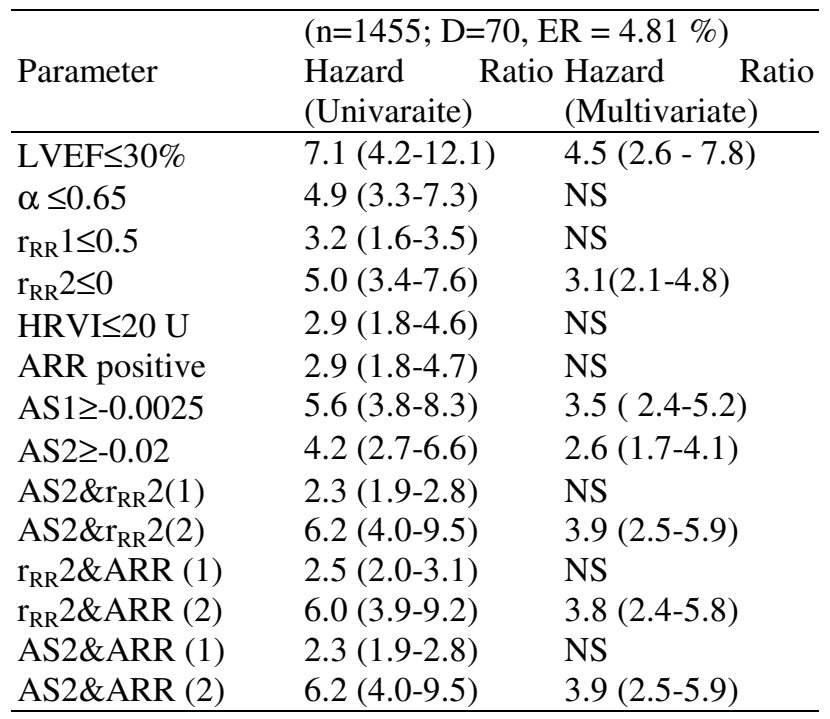

n- number of patients; D - number of deaths, ER - events rate. (1)- one parameter is abnormal; (2) -both

parameters are abnormal

The explorative analysis was performed in patients with moderately reduced LVEF between 30 and $45 \%$. Out of 286 patients in this subgroup 21 (7.34) died. As depicted in the table 3 all analyzed ACF variables were significantly associated with death. The hazard ratio of ACF parameters was higher in this subgroup compared to all patients. The combination of abnormal $\mathrm{r}_{\mathrm{RR}} 2$ and positive arrhythmia on Holter provided very high HR of $=$ 10.0 and PPA of $19 \%$ and was revealed to be strongest predictor for mortality (table 2). Abnormal AS1, combination of abnormal ACF parameters of detrended fluctuations and abnormal short-term fractal exponent $\alpha$ were also revealed to be independent predictors for death in these patients. However, no of clinical covariates, even LVEF and age, analyzed as continuous variable, could be revealed to be predictive for outcome in this subgroup. And again, although slope of ACF of the original RR intervals was predictive for death, the autocorrelation coefficient of original RR intervals was not associated with clinical outcome.

Out of 70 patients with severe depressed LVEF $\square 30 \%$ $15(21.42 \%)$ died. The significant univariate association with mortality in patients with $\mathrm{LVEF}<=30 \%$ was found only by combination of ACF parameters of detrended fluctuations and positive arrhythmia (table 2.).

Table 2. Univariate association of risk variables with death in subgroups

\begin{tabular}{|c|c|c|}
\hline & $\begin{array}{l}\text { LVEF: } 30-45 \\
(\mathrm{n}=286 ; \mathrm{D}= \\
\text { ER }=7.34 \%) \\
\text { Hazard Ratio }\end{array}$ & $\begin{array}{l}\mathrm{LVEF}<=30 \% \\
(\mathrm{n}=70 ; \quad \mathrm{D}=15 ; \\
\mathrm{ER}=21.43 \%) \\
\text { Hazard Ratio }\end{array}$ \\
\hline $\mathrm{r}_{\mathrm{RR}} 2 \leq 0$ & $5.4(2.4-12.4)$ & $1.5(0.7-3.05)$ \\
\hline$\alpha \leq 0.65$ & $5.1(2.4-10.0)$ & $1.5(0.6-4.1)$ \\
\hline $\mathrm{AS} 1 \geq-0.0025$ & $5.2(2.3-11.4)$ & $1.4(0.5-3.9)$ \\
\hline AS2 $\geq-0.02$ & $2.9(1.8-4.8)$ & $0.4(0.1-1.4)$ \\
\hline $\mathrm{AS} 2 \& \mathrm{r}_{\mathrm{RR}} 2(1)$ & $5.3(2.5-11.2)$ & $0.3(0.1-0.9)$ \\
\hline $\mathrm{AS} 2 \& \mathrm{r}_{\mathrm{RR}} 2(2)$ & $6.5(2.7-15.7)$ & $1.4(0.9-2.1)$ \\
\hline $\mathrm{r}_{\mathrm{RR}} 2 \& \mathrm{ARR}$ (1) & $3.2(1.8-5.7)$ & $0.4(0.1-0.9)$ \\
\hline $\mathrm{r}_{\mathrm{RR}} 2 \& \mathrm{ARR}(2)$ & $10.0(3.6-28.0)$ & $1.7(1.0-2.9)$ \\
\hline AS2\&ARR (1) & 4.1(1.9-8.9) & $1.7(0.6-4.4)$ \\
\hline AS2\&ARR (2) & $8.0(2.9-22.2)$ & $1.5(1.1-2.2)$ \\
\hline
\end{tabular}

Out of 70 patients with severe depressed LVEF $\square 30 \%$ $15(21.42 \%)$ died. The significant univariate association with mortality in patients with $\mathrm{LVEF}<=30 \%$ was found only by combination of ACF parameters of detrended fluctuations and positive arrhythmia (table 2.). No of analyzed ECG parameters and clinical covariates were revealed to be predictive for mortality in these patients.

The area under the ROC curve is a measure of accuracy of the parameter to be analyzed. An area of 1 represents a perfect test; an area of $<0.5$ represents a worthless test. According to traditional interpretation of ROC analysis the parameters providing area under ROCcurve between $0.7-0.8$ are fair parameters reliable for performing of diagnose test, the parameters providing area $0.6-07$ are poor and parameters providing area below 0.6 cannot be used for diagnostic propose. As shown in the table $3 \mathrm{r}_{\mathrm{RR}} 2$, AS1, AS2 and $\alpha$ provided area $>0.7$ for all patients.

Table 3 Area under ROC curves

\begin{tabular}{llll}
\hline & All Patients & LVEF: $30-45 \%$ & LVEF $<=30 \%$ \\
\hline $\mathrm{r}_{\mathrm{RR}} 1$ & 0.68 & 0.67 & 0.55 \\
$\mathrm{r}_{\text {RR }} 2$ & 0.72 & 0.74 & 0.54 \\
AS1 & 0.73 & 0.75 & 0.54 \\
AS2 & 0.73 & 0.78 & 0.57 \\
$\alpha$ & 0.75 & 0.73 & 0.57 \\
\hline
\end{tabular}

The area under $\mathrm{r}_{\mathrm{RR}} 2$, AS1, AS2 increased in patients with moderately reduced LVEF, thereby the parameter AS2 showed highest accuracy. In patients with LVEF $\leq$ $30 \%$ the area under all analyzed ROC curves was below 
0.6 indicating poor suitableness of these parameters for risk stratification.

\section{Discussion and conclusions}

In this study ACF parameters were powerful predictors of mortality in post MI patients. We found that autocorrelation coefficient of detrended fluctuations has strong predictive value whereas the autocorrelation coefficient of original Poincare Plot does not predict mortality. This result can be explained by the fact, that $r_{R R}$ of original data assess predominantly long term variability, or in other words, variability of the instantaneous mean of the heart rate, which has more organized behavior than short term fluctuations $\left(\mathrm{r}_{\mathrm{RR}} 1: 0.84\right.$ (63 to 0.90$) ; \mathrm{r}_{\mathrm{RR}} 2: 0.26(-0.03$ to 0.52$\left.) ; \mathrm{p}<0.0001\right)$. This finding is in agreement with previous study [4] described prognostic significance of the short term fractal exponent $\alpha$ provided by DFA. We found very strong correlation between linear autocorrelation coefficient of the detrended fluctuations $\mathrm{r}_{\mathrm{RR}} 2$ and non linear fractal exponent $\alpha(\mathrm{r}=0.95)$. We have also confirmed prognostic significance of the abnormal slope of the lagged ACF of original RR intervals and of detrended fluctuations. Thereby the AS1 was revealed as the strongest predictor. One of possible reasons is the definition of the cut-off of AS2. As shown in the table 4, the area under AS2 is the largest and therefore, by optimized cut-off AS2 should be revealed as strongest predictor in these patients. On the other hand, the difference between areas under AS1 and AS2 is small, so that predictive accuracy of AS1 is also high.

The significant association of frequent ectopic activity with mortality was found in previous works [7] and the univariate association was also confirmed in the present study (table 1). However, frequent ectopy could not be revealed as independent predictor. The hazard ratio provided by VPB frequency alone is clearly lower than by ACF and DFA parameters. However, the abnormal ACF correlates with frequent VPBs. One possible explanation may be that previously defined critical number of VPBs [7] is not actual now due to optimized therapy.

The results of explorative analysis are relevant for the clinical practice. ACF parameters and fractal exponent $\square$, revealed to be independently predictive in patients with moderately reduced LVEF were not predictive in patients with severe left ventricular dysfunction.

Many previous trials assessing predictive value of conventional Holter parameters were performed in patients with wide spectrum of LVEF [1-3]. Although the Holter parameters were revealed to be predictive in these studies the positive predictive accuracy was small. In order to improve the PPA some investigators combined the electrocardiographic parameters with reduced LVEF
[7]. Although this approach improved positive predictive accuracy, it is controversial. It is well known, that mortality and arrhythmic deaths/cardiac arrests occurred more frequently in patients with LVEF $<30 \%$ than in those with LVEF of $30 \%$ to $40 \%$. In this study collective out of 70 patients with LVEF $\leq 30 \% 15$ patients died constituting substantial percentage of $21.43 \%$, in a subgroup with moderately reduced LVEF 21 (7.34\%) patients did not survive follow up period. However, the proportion of patients with severe reduced LVEF is small $(4.81 \%)$, whereas proportion of patients with moderately reduced LVEF is $19.65 \%$ in our patients. In future, the proportion of patients with severe left ventricular dysfunction will be decreased due to progress of therapy. Our approach seems to be appropriate for these patients.

\section{References}

[1] Copie X, Hnatkova K, Staunton A, et al. Predictive power of increased heart rate versus depressed left ventricular ejection fraction and heart rate variability for risk stratification after myocardial infarction. Results of a twoyear follow-up study. J Am Coll Cardiol 1996;27: 270-276

[2] Kleiger RE, Miller JP, Bigger JT et al. Decreased heart rate variability and its association with increased mortality after acute myocardial infarction. Am J Cardiol 1987;59:256262.

[3] La Rovere MT, Bigger JT, Marcus FI, et al. Baroreflex sensitivity and heart-rate variability in prediction of total cardiac mortality after myocardial infarction. ATRAMI (Autonomic Tone and Reflexes After Myocardial Infarction) Investigators. Lancet 1998;351:478-484.

[4] [Huikuri HV, Mäkikallio TH, Peng CK, et al. Fractal correlation properties of $\mathrm{R}-\mathrm{R}$ interval dynamics and mortality in patients with depressed left ventricular function after an acute myocardial infarction. Circulation. 2000;101:47-53.)

[5] Otzenberger H, Gronfier C,.Simon C, et al. Dynamic heart rate variability: A tool for exploring sympathovagal balance continuously during sleep in men. Amer. J. Physiol., 1998; 25: 946-950

[6] Schack B, Grieszbach G. Adaptive Methods of Trend Detection and their Application in Analyzing Biosignals. Biom. J 1994;36:429-452

[7] Moss AJ, Akiyama T. Prognostic significance of ventricular premature beats. Cardiovasc Clin 1974; 6: 273 298

Address for correspondence

Dr. Alexander Berkowitsch

Kerckhoff-Clinic, Beneke Strasse 2-8, 61231, Bad Nauheim, Germany

a.berkowitsch@kerckhoff-klinik.de 